\title{
Lacrimal scintigraphy. I. Compartmental analysis of data
}

\author{
T. E. HILDITCH,${ }^{1}$ C. S. KWOK,${ }^{1 *}$ AND L. A. AMANAT ${ }^{2} \dagger$ \\ From the ${ }^{1}$ Department of Clinical Physics and Bio-Engineering, West of Scotland Health Boards, \\ Glasgow, and the ${ }^{2}$ Tennent Institute of Ophthalmology, University of Glasgow, Western Infirmary, Glasgow
}

SUMMARY Linear compartmental analysis was applied to lacrimal scintigraphy data from asymptomatic and obstructed lacrimal systems. Failure to achieve satisfactory results in most of the asymptomatic systems studied suggests that variable tear flow is a feature of normal lacrimal drainage which precludes linear analysis. However, in cases where the analysis was successful the results were consistent with the occurrence of reflux between the conjunctival and lacrimal sacs, and between the lacrimal sac and nasolacrimal duct. Further support for the existence of reflux between compartments was provided by the analysis of obstructed systems, particularly those with obstruction at the upper end of the nasolacrimal duct. In such cases there was rapid equilibrium of the tracer between the conjunctival and lacrimal sacs, which in the absence of tear flow could be explained by reflux between these 2 compartments.

Because of its physiological character lacrimal scintigraphy (LS) has been considered a suitable technique for studying the dynamics of lacrimage drainage. ${ }^{1}$ Carlton et al. ${ }^{2}$ introduced the concept of quantitation by measuring the transit time of the tracer to the lacrimal sac and nasolacrimal duct, and in a later study ${ }^{3}$ used the 'region of interest' technique to plot the activity-time function curves of the various compartments of the lacrimal drainage system (LDS) using a computer. Similar studies were carried out by other investigators, ${ }^{45}$ who also measured the $T_{1 / 2}$ values for the conjunctival sac. The analysis of lacrimal scintigraphy data was extended by Hurwitz et al. ${ }^{6}$ to include the transit times $\left(\mathrm{t}_{\max }\right)$ and $\mathrm{T}_{1 / 2}$ values for the various compartments of the LDS, while S申rensen and Jensen ${ }^{7}$ used a simple compartmental model of the conjunctival sac to estimate the fractional turnover rate of tears to measure the tear flow. The results to date have shown that, although the more elaborate analysis has thrown further light on the physiology of lacrimal drainage, it would appear to

*Present address: Department of Physics, Hamilton Clinic, Hamilton, Ontario, Canada.

†Present address: District General Hospital, Lowestoft Road, Gorleston, Great Yarmouth.

Correspondence to Dr T. E. Hilditch, Department of Clinical Physics and Bio-Engineering, 11 West Graham Street, Glasgow G4 9LF. have contributed little to the clinical value of LS, since in the majority of patients with obstructive epiphora an accurate diagnosis of the cause can be made by examining the serial images in conjunction with the dynamic curves alone. ${ }^{8}$

However, in a study of asymptomatic lacrimal systems ${ }^{9}$ the authors found that in a significant number of these systems the nasolacrimal duct offered a physiological obstruction to tear flow, which suggested that LS had no role in the clinical evaluation of tear passage through the nasolacrimal duct to the nose. These observations would therefore imply that, because the lacrimal drainage system does not empty at a constant rate, it would be difficult to analyse LS data and establish quantitative criteria on the basis of a linear flow, as has been done in the other studies.

The present paper describes an attempt to analyse LS data by means of a multicompartment model to explore in more detail the dynamics of lacrimal drainage. By introducing this more complicated model instead of the simple model used by S申rensen and Jensen ${ }^{7}$ it was our intention to determine whether reflux can occur between various compartments and whether there is any need to postulate a variable flow as other workers had done ${ }^{10}$ in their experiments. The analysis was applied to systems with no obstruction and to systems with an obstruction at differing levels. 


\section{Material and methods}

The initial aim was to perform compartmental analysis on a group of asymptomatic systems to obtain normal values of the various parameters associated with lacrimal drainage, so that these could be compared with estimates in obstructed systems. However, for reasons which are given later, this proved not to be a practical proposition. Indeed, out of the 20 asymptomatic systems selected for study compartmental analysis could be performed satisfactorily in only 2 . Nine systems within this group were also studied with a view to determining the exponential components of tracer disappearance from the conjunctival sac. Twelve other symptomatic systems with a demonstrable obstruction at the lower end of the nasolacrimal duct, proved equally difficult to analyse. In fact only 2 of these could be analysed. However, analysis was performed satisfactorily in 10 systems in which an obstruction was present at the upper end of the nasolacrimal duct-that is, where only two compartments, representing the conjunctival and lacrimal sacs, needed analysis. Also analysed were 11 systems with complete canalicular obstruction-that is, where only one compartment, the conjunctival sac, was present. Thus altogether 25 systems were studied by multicompartment analysis.

Data were acquired with an Ohio Nuclear Series 100 gamma camera linked to a Varian V76 computer. Subjects were positioned upright with the cornea at a distance of $85 \mathrm{~mm}$ from the hole of the $2.5 \mathrm{~mm}$ pinhole collimator. The study commenced with the simultaneous instillation of $2 \mathrm{MBq}^{99 \mathrm{~m}} \mathrm{Tc}$ tin colloid in $15 \mu \mathrm{l}$ isotonic saline in each conjunctival sac with micropipettes. Data were collected for the next 10 minutes in the form of a series of 100 dynamic frames each of $6 \mathrm{~s}$ duration and stored in the computer. A summed view of these frames was produced at the end of the study to enable regions of interest to be defined. These consisted of the conjunctival sac, lacrimal sac, nasolacrimal duct, and nasal cavity. Curves representing the time course of radioactivity in these compartments, corrected for radioactive decay, were generated by the computer.

Before compartmental analysis was performed the original data from each compartment were corrected for the increase in counting sensitivity that occurs as the radioactive tracer moves towards the centre of the object plane. This is a feature of a pinhole type of collimator, and in the present system the counting sensitivity for a source at the lateral canthus of the eye was typically $30 \%$ less than at the inner edge of the lacrimal sac. Thus correction was made to the acquired count at each point in the image matrix by normalising the counting sensitivity to that at the centre of the object plane. Analysis was performed

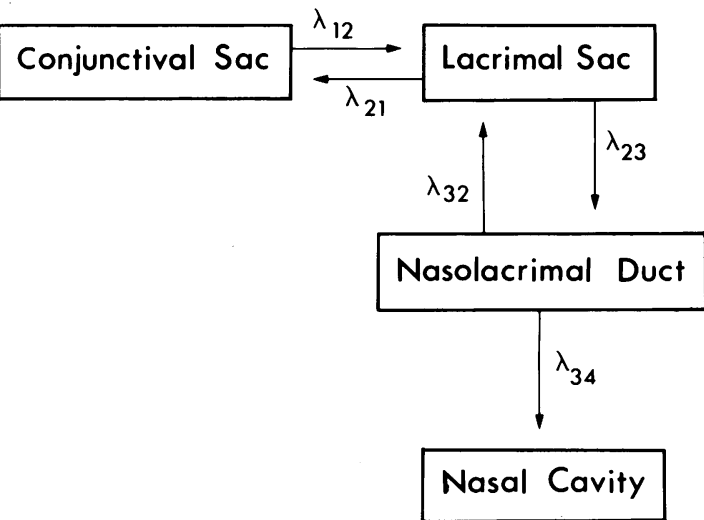

Fig. 1 Four-compartment model used in the analysis of lacrimal scintigraphy data. The object of the analysis is to evaluate the fractional transfer coefficients between each compartment.

on the corrected time/radioactivity curves for each compartment.

The model used in the analysis consists of 4 compartments ${ }^{11}$ representing the conjunctival sac, the lacrimal sac, the nasolacrimal duct, and the nasal cavity respectively (Fig. 1). As indicated in the diagram, allowance is made for possible reflux between the first and second compartments and between the second and third compartments. Loss of tracer from the conjunctival sac by routes other than the canaliculi was neglected on the basis of findings which are presented later. If $\lambda_{i j}$ is the fractional transfer coefficient between each compartment-i.e., the fraction of the contents of compartment $i$ transferred to compartment $j$ per unit time - then the rate of change $q_{i}$ (the amount of tracer in compartment $i$ at any time) is given by

$$
\frac{\mathrm{dq}_{\mathrm{i}}}{\mathrm{dt}}=-\mathrm{q}_{\mathrm{i}} \sum_{\mathrm{i} \neq \mathrm{j}} \lambda_{\mathrm{ij}}+\sum_{\mathrm{i} \neq \mathrm{j}} \lambda_{\mathrm{ji}} \mathrm{q}_{\mathrm{j}}
$$

The method of determining the $\lambda_{i j}$ followed a standard approach readily performed on a digital computer ${ }^{11}$ and makes use of the fact that $\mathrm{q}_{i}$ is known for each compartment. It consists of estimating the changes in $\mathrm{q}_{i}$ for a given compartment over a series of 50 consecutive time intervals of $12 \mathrm{~s}$ and noting the values of $\mathrm{q}_{\mathrm{i}}$ at these times in neighbouring compartments. This produces for each compartment a set of 50 overdetermined linear equations with unknowns $\lambda_{i j}$. Least sum of squares estimates of the $\lambda_{i j}$, along with standard errors, were obtained from these equations by linear regression analysis. ${ }^{12}$ Only those estimates with a magnitude greater than 2 standard errors were considered significant. 


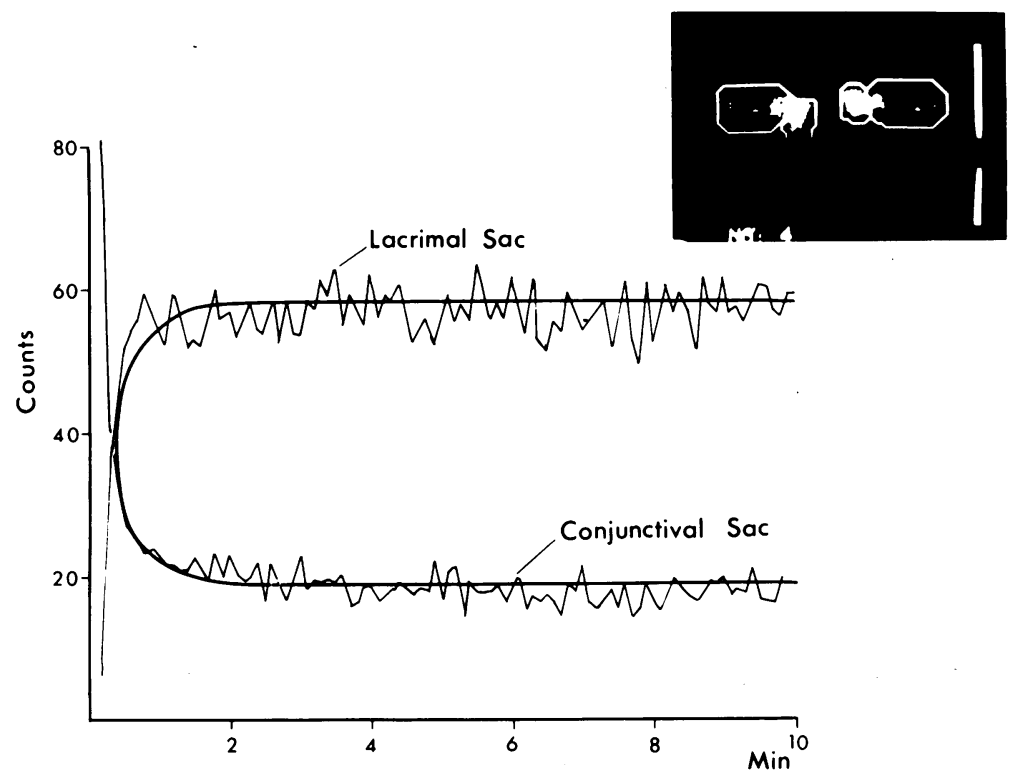

Fig. 2 Results from a system with obstruction at the lower end of the lacrimal sac. The smooth curves represent the best fits to the observed data for the conjunctival and lacrimal sacs.

\section{Results}

In only 2 out of 11 systems studied with canalicular obstruction was there any significant loss of tracer (to tissues outside the lacrimal drainage system) in the 10-minute study period. The mean estimated fractional loss rate in these 2 systems was $0.018 \mathrm{~min}^{-1}$ (Table 1), which is negligible. This gave justification to ignoring any transconjunctival loss of tracer in the compartmental analysis. The results of the analysis in all the 25 systems studied are given in Table 1 , which shows the fractional transfer coefficients for the various compartments in the different types of obstruction. Fig. 2 shows an example of a system with an obstruction at the lower end of the lacrimal sac. It can be seen that the computed curves based on the estimated transfer coefficients seem to be good fits to the observed data. Figs. 3 and 4 are examples of a

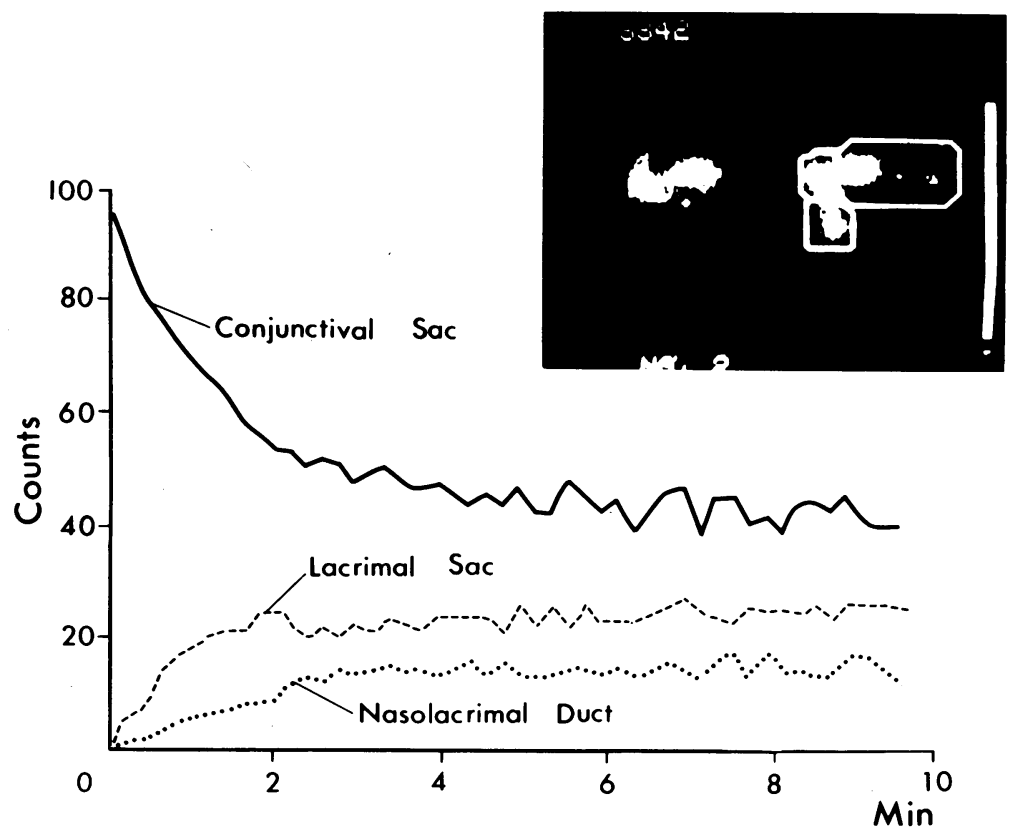

Fig. 3 Results from a system with obstruction at the distal end of the nasolacrimal duct. The quantity of radioactive tracer in each compartment has reached an equilibrium value by the end of the study period. 
Table 1 Results of compartmental analysis showing mean ( $\pm S D)$ fractional transfer coefficients $\left(\mathrm{min}^{-1}\right)$

\begin{tabular}{|c|c|c|c|c|}
\hline Level of obstruction & $\begin{array}{l}\text { Common canalicular } \\
\text { obstruction } \\
\text { (1 compartment) }\end{array}$ & $\begin{array}{l}\text { Obstruction at upper } \\
\text { end of } N L D \\
\text { ( } 2 \text { compartments) }\end{array}$ & $\begin{array}{l}\text { Obstruction at lower } \\
\text { end of NLD } \\
\text { (3 compartments) }\end{array}$ & $\begin{array}{l}\text { No } \\
\text { obstruction } \\
\text { (4 compartments) }\end{array}$ \\
\hline $\begin{array}{l}\text { Number of systems studied } \\
\text { Mean transfer coefficient from }\end{array}$ & 11 & 10 & 2 & 2 \\
\hline $\begin{array}{l}\text { conjunctival sac } \\
\text { Mean transfer coefficient from }\end{array}$ & $0 \cdot 018 \pm 0 \cdot 017^{*}$ & - & 一 & - \\
\hline $\begin{array}{l}\text { conjunct. sac to lac. sac } \\
\text { Mean reflux coefficient from lac. }\end{array}$ & - & $1 \cdot 68 \pm 1 \cdot 92$ & $1 \cdot 14 \pm 1 \cdot 14$ & $3 \cdot 24 \pm 1 \cdot 98$ \\
\hline $\begin{array}{l}\text { sac to conjunct. sac } \\
\text { Mean transfer coefficient from lac. }\end{array}$ & - & $0.78 \pm 0.54$ & $0 \cdot 66 \pm 0.36$ & $0 \cdot 72 \pm 0 \cdot 60$ \\
\hline $\begin{array}{l}\text { sac to nasolac. duct } \\
\text { Mean reflux coefficient from nasolac. }\end{array}$ & - & - & $0 \cdot 30 \pm 0 \cdot 06$ & $0.90 \pm 0.66$ \\
\hline $\begin{array}{l}\text { duct to lac. sac } \\
\text { Mean transfer coefficient from nasolac. }\end{array}$ & - & - & $0.54 \pm 0.24$ & $0 \cdot 36 \pm 0 \cdot 12$ \\
\hline duct to nose & - & - & - & $0 \cdot 18 \pm 0 \cdot 12$ \\
\hline
\end{tabular}

${ }^{*}$ Only 2 systems showed loss of tracer from the conjunctival sac. Lac. = lacrimal. Conjunct. $=$ conjunctival.

system with an obstruction at the distal end of the nasolacrimal duct and a system with no obstruction respectively.

In 7 out of 9 systems with no evidence of obstruction there were 2 exponential components in the tracer disappearance curve for the conjunctival sac, as determined by an exponential curve stripping routine. The rate constant of the first component, the fast component, had a mean value of $4.61 \mathrm{~min}^{-1}$ and the second, slower component had a mean value of $0.063 \mathrm{~min}^{-1}$ (Table 2). These 2 phases of tracer disappearance are clearly seen in the curve for conjunctival sac in Fig. 4, where $50 \%$ of the initial radioactivity has left the conjunctival sac well within the first minute. The 2 remaining cases with no evidence of obstruction demonstrated only one component of tracer disappearance from the first compartment; in one case loss of tracer took place rapidly, while in the other the clearance was much slower (Table 2).

An example of an unobstructed system where the flow pattern was variable is given in Fig. 5 . The curves are consistent with a sudden increased clearance of tracer from the lacrimal sac towards the end of the study period.

\section{Discussion}

At first sight it might be concluded that the experimental data from a dynamic radionuclide investiga-
Fig. 4 Results from a system showing continuous drainage of tracer from each compartment. Tracer can be seen in the nasal cavity within 1 minute.

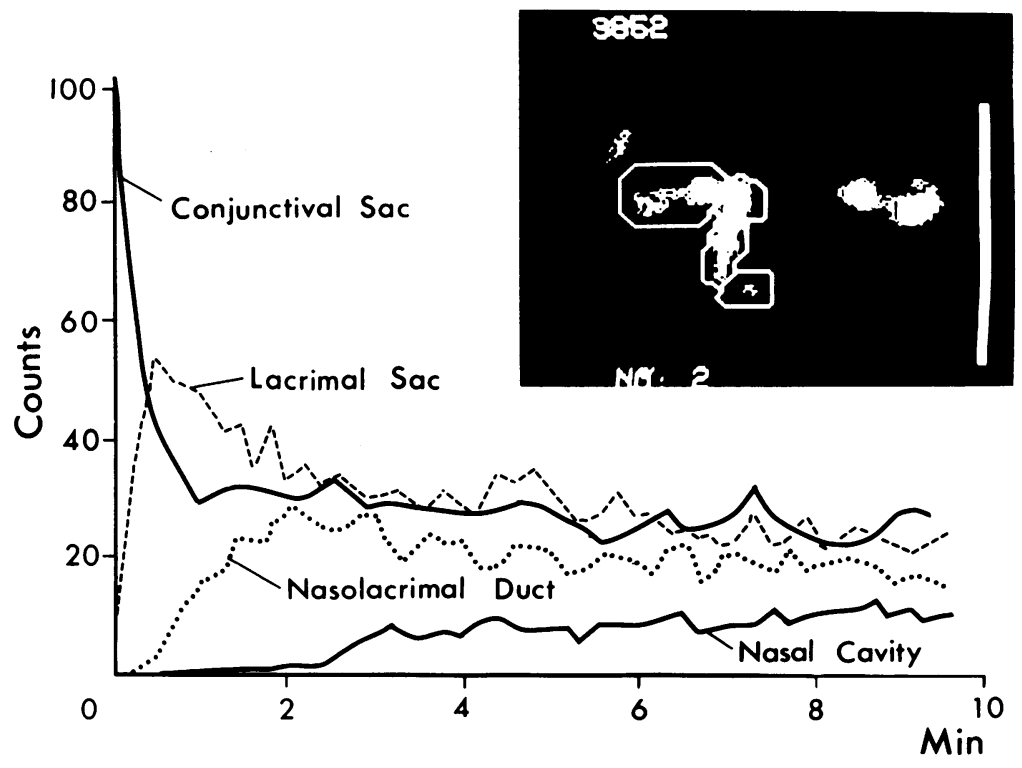




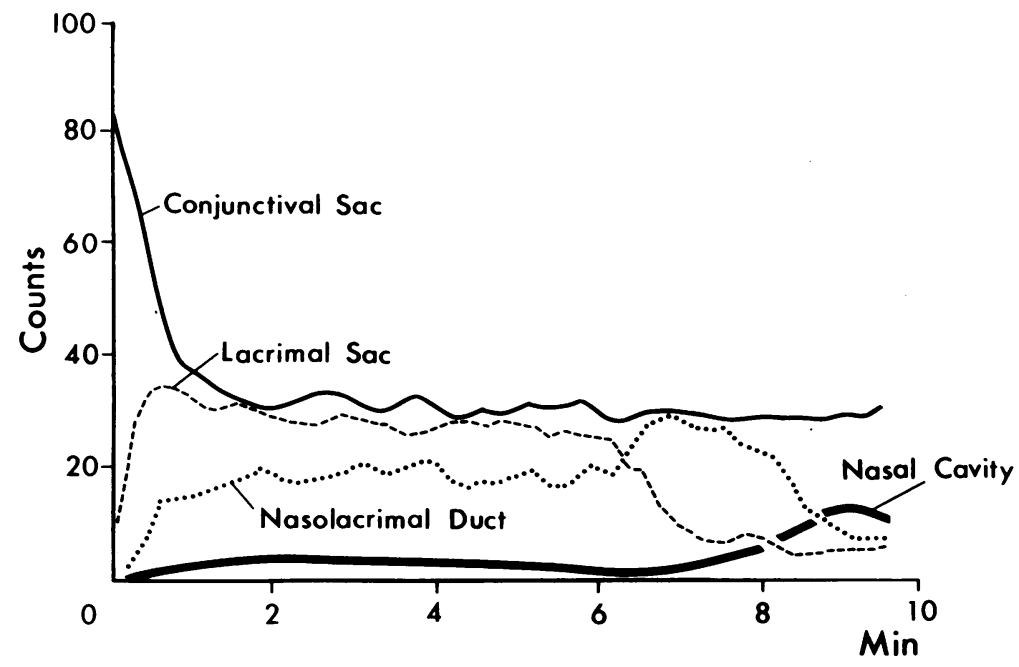

Fig. 5 Results from a system where the flow of tracer was variable. A sudden release of tracer from the lacrimal sac can be seen towards the end of the study period.

tion of the lacrimal drainage system are ideally suited for linear compartmental analysis with reflux between compartments. However, problems were encountered at the outset of the present work when asymptomatic systems with no clinical evidence of obstruction were studied to establish normal values for the various parameters of a 4-compartment linear model.

First, approximately $25 \%$ of the asymptomatic systems selected for study manifested apparent obstruction by the end of the 10-minute study period at either the upper or, more often, the lower end of the nasolacrimal duct. These are similar to the findings of Chavis et al. ${ }^{9}$ where no tracer was detected in the nasal cavity in $32 \%$ of normal asymptomatic systems by the end of a 12-minute study period. In all the present cases the apparent obstruction was cleared by the repeated instillation of $15 \mu \mathrm{l}$ drops of saline at 1-minute intervals. However, in such cases lacrimal drainage in the normal course of events was likely to be nonlinear, and thus these cases had to be excluded from the compartmental analysis. Secondly, within the remaining asymptomatic cases there were some where the clearance of tracer manifested definite nonlinearity within the 10 -minute study period (Fig. 5). The findings in these cases suggest that, while there was probably a small but steady flow of tear fluid through the system, this could be augmented periodically by sudden increases in flow due to

Table 2 Analysis of time activity curves for conjunctival sac in 9 systems with 4 compartments present

\begin{tabular}{lll}
\hline $\begin{array}{l}\text { Number of } \\
\text { systems }\end{array}$ & $\begin{array}{l}\text { Fast component }\left(\text { min }^{-1}\right) \\
(\text { mean } \pm S D)\end{array}$ & $\begin{array}{l}\text { Slow component }\left(\text { min }^{-1}\right) \\
(\text { mean } \pm S D)\end{array}$ \\
\hline 7 & $4.61 \pm 1.58$ & $0.063 \pm 0.036$ \\
1 & 3.316 & - \\
1 & - & 0.065 \\
\hline
\end{tabular}

pressure build-up within the lacrimal sac and nasolacrimal duct. Systems manifesting such features could not be included in the compartment analysis. Finally, even within those cases where the flow of tracer between compartments appeared to be linear, and where the tracer had reached the nasal cavity before the end of the study, difficulties were found in obtaining consistent results in the analysis procedure. This was in spite of the fact that correction had been made to the observed data for counting sensitivity variations which if left uncorrected would have affected the analysis.

One possible source of difficulty might be the transconjunctival loss of tracer, which was ignored in the analysis. S申rensen and Jensen ${ }^{13}$ reported a significant loss of tracer through the conjunctiva in their study using ${ }^{99 \mathrm{~m}} \mathrm{Tc}$ pertechnetate, but ${ }^{99 \mathrm{~m}} \mathrm{Tc}$ tin colloid was employed in the present study. The studies in subject with canalicular obstruction would suggest that with the tin colloid there is little transconjunctival loss (Table 1), and there would appear to be a justification for neglecting it. It is also unlikely that adherence of the tracer to the surfaces in the lacrimal drainage system is a problem, since Murai ${ }^{10}$ has shown that ${ }^{99 \mathrm{~m}} \mathrm{Tc}$ pertechnetate does not adhere to the mucous membrane lining the lacrimal passage. Indeed, other studies ${ }^{14}$ have shown that colloidal tracer is less likely to adhere to a surface than ionic pertechnetate. Perhaps a more probable source of error is the difficulty encountered in practice in separating the compartments, particularly the nasolacrimal duct from the lacrimal sac, when the regions of interests are defined on the computer display (Fig. 6). This could lead to overlap of the regions of interest, resulting in distortion of the dynamic curves for each compartment. The magnitude of this problem, however, is dependent on the resolution of the 
Fig. 6 Scintigraph from a patient where there was difficulty in separating the lacrimal and conjunctival sacs.

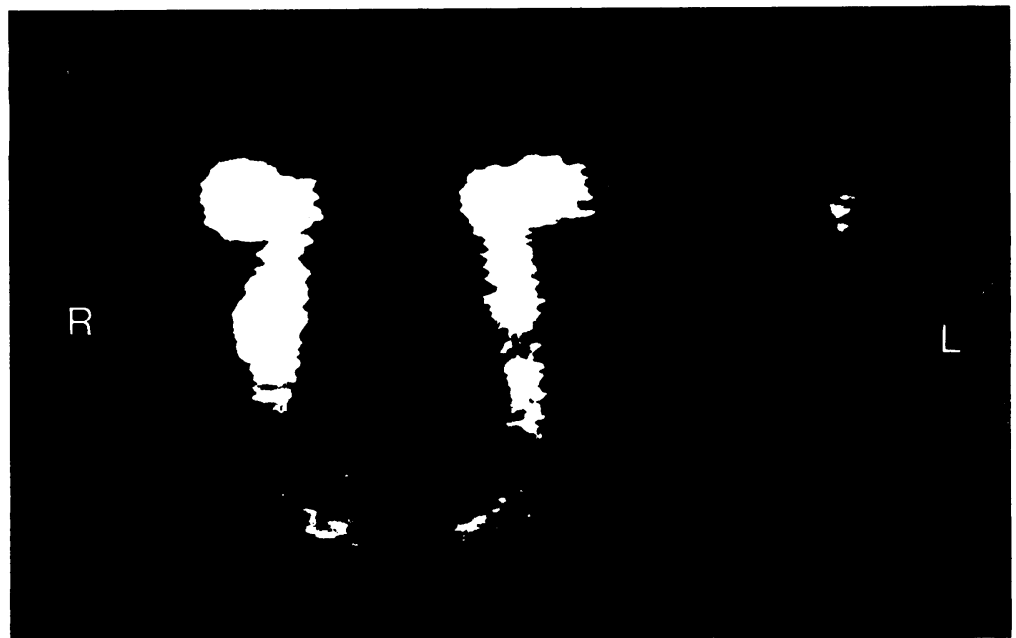

imaging system used in the study. Any improvements in resolution would reduce the difficulty in separating the compartments.

A more fundamental problem, however, is the underlying assumption that the flow of tears is indeed constant when the transfer of tracer between compartments appears to be occurring at a steady rate (Fig. 4). If there is a short period of increased tear flow due to irritation of the conjunctiva at the moment of instillation of the tracer, this would also produce time radioactivity curves similar to those shown in Fig. 4. In fact the present analysis of curves from the conjunctival sac into exponential components has confirmed the apparent existence of 2 phases of clearance, a finding that has been interpreted as indicating early increased flow. ${ }^{7}$ There is good agreement between the mean rate constant of the slow component in the present series, $0.063 \pm$ $0.036 \mathrm{~min}^{-1}$ and a previously published value of $0.088 \pm 0.018 \mathrm{~min}^{-1} .^{7}$ Both sets of values compare reasonably with a recent estimation of $0 \cdot 12 \pm 0.03$ $\min ^{-1}$ for the mean fluorescein turnover rate. ${ }^{15}$ It is inappropriate, however, to compare the estimated rate constants for the first component because an exponential curve stripping routine was used in the present work, whereas S $\phi$ rensen and Jensen ${ }^{7}$ simply determined slopes from a $\log /$ linear plot of the data, and because the sensitivity correction in the present procedure enhances the early part of the curve from the conjunctival sac.

Thus the present observations in asymptomatic lacrimal systems would suggest that variable tear flow is a normal feature of lacrimal drainage. Such systems cannot be described adequately by a linear compartmental model. In some cases, however, it may be possible to analyse the observed data on the basis of this type of model. The results in 2 cases in which and lacrimal sacs and between the lacrimal sac and

Further information on the dynamics of lacrimal drainage, in particular on the question of reflux,${ }^{16} \mathrm{can}$ be gleaned from the present studies of obstructed systems. The analysis of 2-compartment systemsi.e., where there was obstruction at the upper end of the nasolacrimal duct-clearly points to the existence of reflux between the conjunctival and lacrimal sacs. This would appear to be the most likely explanation for the rapidity with which equilibrium can be reached between the 2 compartments (Fig. 2), considering there is no net fluid flow through such systems. The administered radioactive tracer is simply mixed uniformly throughout the system by the lacrimal pump, which presumably is still functional. Furthermore, the compartmental analysis of 3 compartment systems-i.e., where there was obstruction at the lower end of the nasolacrimal duct-revealed, albeit in only 2 systems, that there lacrimal sac (Table 1). Thus the present work has demonstrated that lacrimal drainage is a complex compartments being possible. It does not lend itself easily to simple analysis.

In spite of these problems it is interesting to consider whether lacrimal scintigraphy can provide some indication of the magnitude of tear flow. On the assumption that the slow component of clearance from the conjunctival sac represents the net fractional turnover of that compartment, and taking a compartmental volume of $7 \mu \mathrm{l},{ }^{15}{ }^{17}$ a mean tear flow of $0.45 \mu \mathrm{l}$ per min can be estimated from the present data (Table 2 ). This compares well with a value of $0.6 \mu \mathrm{l}$ per min analysis was carried out satisfactorily are consistent with the occurrence of reflux between the conjunctival nasolacrimal duct (Table 1). can be reflux between the nasolacrimal duct and the process with variable tear flow and reflux between 
published by S $\phi$ rensen and Jensen, ${ }^{17}$ who used the same method of calculation. An alternative method of calculating tear flow is based on the compartmental model of Fig. 1. Net flow through the system is given by $V_{1} \lambda_{12}-V_{2} \lambda_{21}$ where $V_{1}$ and $V_{2}$ are the volumes of the conjunctival and lacrimal sacs respectively. Taking $V_{1}$ as $7 \mu l$ and estimating $V_{2}$ to be $20-30 \mu l$ from published anatomical data, ${ }^{18}$ and taking $\lambda_{12}$ and $\lambda_{21}$ to be 3.24 and $0.72 \mathrm{~min}^{-1}$ respectively (Table 1), a tear flow of 1-8 $\mu \mathrm{l}$ per min can be estimated. These estimates of tear flow of course depend very much on the estimated volumes of each compartment, but they give order of magnitude results which compare favourably with the published range of $0 \cdot 2-20 \mu \mathrm{l}$ per min as reviewed by Sørensen and Jensen. ${ }^{17}$

We express our thanks to the technical staff of the Nuclear Medicine Department, Western Infirmary, Glasgow, for their help and cooperation in carrying out this study, and Mrs R. Brown for her invaluable secretarial assistance and for typing the manuscript.

\section{References}

1 Rossomondo RM, Carlton, WH, Trueblood JH, Thomas RP. A new method of evaluating lacrimal drainage. Arch Ophthalmol 1972; 88: 523-5.

2 Carlton WH, Trueblood JH, Rossomondo RM. Clinical evaluation of microscintigraphy of the lacrimal drainage apparatus. $J$ Nucl Med Allied Sci 1973; 14: 89-92.

3 Trueblood JH, Rossomondo RM, Carlton WH, Wilson LA. Corneal contact times of ophthalmic vehicles-evaluation by microscintigraphy. Arch Ophthalmol 1975; 93: 127-30.
4 Hardberger R, Hanna C, Boyd CM. Effect of drug vehicles on ocular contact time. Arch Ophthalmol 1975; 93: 42-5.

5 Denffer HV, Dressler J. Radionuclide dacryocystography for demonstrating obstructions of the lacrimal drainage system. Albrecht von Graefes Arch Klin Ophthalmol 1964; 191: 321-8.

6 Hurwitz JJ, Maisey MN, Welham RAN. Quantitative lacrimal scintillography. 1. Method and physiological application. Br J Ophthalmol 1975; 59: 308-12.

7 S $\phi$ rensen T, Jensen FT. Methodological aspects of tear flow determination by means of a radioactive tracer. Acta Ophthalmol (Kbh) 1977; 55: 726-38.

8 Amanat LA, Wraight EP, Watson PG, Hawkins TD. Role of lacrimal scintigraphy and subtraction macrodacryocystography in the management of epiphora. Br J Ophthalmol 1979; 63: 511-9.

9 Chavis RM, Welham RAN, Maisey MN. Quantitative lacrimal scintillography. Arch Ophthalmol 1978; 96: 2066-8.

10 Murai Y. Studies of tear flow using sodium pertechnetate $\mathrm{Tc}^{99 \mathrm{~m}}$ Jpn J Ophthalmol 1976; 20: 283-9.

11 Jacquez JA. Compartmental analysis in biology and medicine. Amsterdam, London, New York: Elsevier, 1972.

12 Davies OL, Goldsmith PL. Statistical methods in research and production. Edinburgh: Oliver and Boyd, 1972.

13 S $\phi$ rensen T, Jensen FT. Conjunctival transport of technetium99m pertechnetate. Acta Ophthalmol (Kbh) 1979; 57: 691-9.

14 Moore PH, Mettler FA. Skin decontamination of commonly used medical radionuclides. J Nucl Med Allied Sci 1980; 21: 475-6.

15 Jordan A, Baum J. Basic tear flow. Ophthalmologica 1980; 87: 920-30.

16 Welham RAN. The watery eye. Br J Hosp Med 1975; 13: 692-700.

17 S $\phi$ rensen T, Jensen FT. Tear flow in normal human eyes. Determination by means of radioisotope and gamma camera. Acta Ophthalmol (Kbh) 1979; 57: 564-81.

18 Jones LT. An anatomical approach to problems of the eyelids and lacrimal apparatus. Arch Ophthalmol 1961; 66: 111-24. 